\title{
Deep sequencing of small RNAs reveals the repertoire of miRNAs and piRNAs in Biomphalaria glabrata
}

\author{
Fábio Ribeiro Queiroz, Laysa Gomes Portilho², Wander de Jesus Jeremias, Élio Hideo Babá1, \\ Laurence Rodrigues do Amaral2,6, Luciana Maria Silva ${ }^{4}$, Paulo Marcos Zech Coelho', \\ Roberta Lima Caldeira ${ }^{5}$, Matheus de Souza Gomes ${ }^{2,6 /+}$ \\ ${ }^{1}$ Fundação Oswaldo Cruz-Fiocruz, Instituto René Rachou, Grupo de Pesquisa em Biologia do Schistosoma mansoni e sua Interação \\ com o Hospedeiro, Belo Horizonte, MG, Brasil \\ ${ }^{2}$ Universidade Federal de Uberlândia, Laboratório de Bioinformática e Análises Moleculares, Patos de Minas, MG, Brasil \\ ${ }^{3}$ Universidade Federal de Ouro Preto, Escola de Farmácia, Departamento de Farmácia, MG, Brasil \\ ${ }^{4}$ Fundação Ezequiel Dias, Serviço de Biologia Celular do Departamento de Pesquisas e Desenvolvimento, Belo Horizonte, MG, Brasil \\ ${ }^{5}$ Fundação Oswaldo Cruz-Fiocruz, Instituto René Rachou, Grupo de Pesquisa em Helmintologia e Malacologia Médica, \\ Belo Horizonte, MG, Brasil \\ ${ }^{6}$ Universidade Federal de Uberlândia, Rede Multidisciplinar de Pesquisa, Ciência e Tecnologia, Patos de Minas, MG, Brasil
}

BACKGROUND Biomphalaria glabrata snails are widely distributed in schistosomiasis endemic areas like America and Caribe, displaying high susceptibility to infection by Schistosoma mansoni. After the availability of B. glabrata genome and transcriptome data, studies focusing on genetic markers and small non-coding RNAs have become more relevant. The small RNAs have been considered important through their ability to finely regulate the gene expression in several organisms, thus controlling the functions like cell growth, metabolism, and susceptibility/resistance to infection.

OBJECTIVE The present study aims on identification and characterisation of the repertoire of small non-coding RNAs in $B$. glabrata (Bgl-small RNAs).

METHODS By using small RNA sequencing, bioinformatics tools and quantitative reverse transcription polymerase chain reaction (RT-qPCR), we identified, characterised, and validated the presence of small RNAs in B. glabrata.

FINDINGS 89 mature miRNAs were identified and five of them were classified as Mollusk-specific. When compared to model organisms, sequences of $B$. glabrata miRNAs showed a high degree of conservation. In addition, several target genes were predicted for all the mature miRNAs identified. Furthermore, piRNAs were identified in the genome of $B$. glabrata for the first time. The $B$. glabrata piRNAs showed strong conservation of uridine as first nucleotide at 5 ' end, besides adenine at 10 th position. Our results showed that $B$. glabrata has diverse repertoire of circulating ncRNAs, several which might be involved in mollusk susceptibility to infection, due to their potential roles in the regulation of $S$. mansoni development.

MAIN CONCLUSIONS Further studies are necessary in order to confirm the role of the Bgl-small RNAs in the parasite/host relationship thus opening new perspectives on interference of small RNAs in the organism development and susceptibility to infection.

Key words: schistosomiasis - non-coding RNAs - mollusk vectors - next-generation sequencing

The Biomphalaria sp glabrata snails are the most important intermediate hosts in the transmission of Schistosoma mansoni, one of the main causative agents of hepatosplenic form of schistosomiasis in America and Caribe. The disease affects millions of people worldwide and, in Brazil its transmission is maintained mainly by B. glabrata, which has a wide geographic distribution in endemic areas, showing high susceptibility to infection by $S$. mansoni. (1) The interaction between parasite and mollusk is complex and well described in many aspects. (2) However, the genetic mechanisms that closely explain

doi: 10.1590/0074-02760190498

Financial support: FAPEMIG (project numbers: APQ-00467-13, APQ01179-15 and APQ-01766-15), CNPq (project numbers: 479890/2013-7, 308869/2017-6, 306053/2017-9 and 427855/2018-7), Instituto René Rachou, FIOCRUZ, Fundação Ezequiel Dias. This study was financed in part by the CAPES (Finance Code 001)

FRQ and LGP contributed equally to this work.

+Corresponding author: matheusgomes@ufu.br

(1) https://orcid.org/0000-0001-7352-3089

Received 23 December 2019

Accepted 22 May 2020 this interaction are poorly understood. The habits of the human population, the snail distribution, and the absence of basic sanitation, make schistosomiasis very difficult to be eradicated through the interruption of the parasite life cycle. Furthermore, one of the factors that influence the susceptibility of the snail to infection by $S$. mansoni, is the activity of its internal defense system (IDS), which relies on hemocytes and soluble components of hemolymph. ${ }^{(3,4)}$ Studies showed that transferring the hemolymph from snails resistant to $S$. mansoni infection to susceptible ones increases the resistance of the later. ${ }^{(5)}$ These results brought to light the question whether is small RNA part of the genetic mechanisms involved in controlling the activity of the hemocytes, and soluble factors of hemolymph, which are determinants of the susceptibility/resistance of the snail to infection by the trematode.

After the availability of the genome and the transcriptome data of $B$. glabrata ${ }^{(6)}$ the studies with focus on genetic markers have become more relevant. Our research group identified genes from the small RNA pathway of different developmental stages of B. glabrata, and changes on their expression profiles during 
the infection by $S$. mansoni. ${ }^{(7)}$ The miRNA expression profile and its pathway have been extensively described in S. mansoni ${ }^{(8,9,10)}$ but in B. glabrata the studies directed towards the identification and characterisation of these small RNAs are still poorly explored.

Small RNAs and their silencing pathways have been considered important in several organisms, since they orchestrate gene expression through a fine and specific regulation process. ${ }^{(11)}$ Some of the most important small RNAs are microRNAs (miRNAs) and PIWI-interacting RNAs (piRNAs), which differ in the number of nucleotides, biogenesis pathway, biological origin and functions, and target genes. Typically, miRNAs have 17 to 24 nucleotides, while in piRNAs, the nucleotides range between 23 to 35.The miRNAs are the best-characterised class among all small RNAs, and their sequences and features can be found in the main database containing thousands of miRNAs from several species (miRBase - $\mathrm{http}$ :// www.mirbase.org/). Another class of endogenous small RNAs, piRNAs, are longer and their active sequences are more diverse when compared to miRNAs. The sequences and features of piRNAs are studied using databases like piRNABank (http://pirnabank.ibab.ac.in/) and piRBase (http://regulatoryrna.org/database/piRNA).

The biogenesis of miRNAs is well studied, and it relies on nuclear and cytoplasmic proteins. Normally, the mature miRNAs recognise the mRNA targets 3' UTR region and the pairing does not need to be perfect for silence activity. ${ }^{(12)}$ The association between these molecules causes blockade of translation or mRNA degradation, reducing this way levels of the protein coded by a specific gene target. ${ }^{(12)}$ The piRNAs can be originated from transposable elements and genomic clusters and, their main functions are closely related to transposons control, mainly on germ line cells. ${ }^{(13,14,15)}$ However, it is a currently well-known fact that piRNAs are involved in the development of somatic tissues such as, neurons, muscle and adult stem cells. ${ }^{(16,17)}$ The piRNAs have a notable diversity in sequences, except in the first nucleotide at 5' end, frequently one uridine, beyond an adenine on the tenth position. ${ }^{(13)}$ The biogenesis of the piRNA is not well known as that of miRNAs, and it can be divided into two steps: transcription of long precursors inside the nucleus, followed by their exportation to the cytoplasm, where it is processed to generate mature piRNAs. The mature piRNAs associated with Piwi proteins act on repression of translation or cleavage of mRNAs targets. ${ }^{(18)}$ In animals, besides the canonical biogenesis of the piRNAs, another common pathway is the "ping-pong cycle", that further shapes the piRNA population by amplifying sequences targeting active transposons. ${ }^{(13)}$ On the cytoplasm, precursors of piRNAs exported from nucleus are processed without dicer and in association with AGO-like proteins, act to control gene expression and transposons activities. ${ }^{(19)}$

Advances in high-throughput sequencing technologies have significantly increased the understanding of the role of small RNAs on several species, including $B$. glabrata. The genome and transcriptome sequencing ${ }^{(6)}$ revolutionised the knowledge of the relationship between the snail and $S$. mansoni by bringing to light the existence of processing machinery of small RNAs such as miRNAs and piRNAs, in B. $_{\text {glabrata }}{ }^{(6,7)}$ However, it is necessary to identify and characterise the profile of miRNAs and piRNAs expression within this organism. In the present work our group applied high-throughput sequencing technologies and bioinformatics tools to reveal the expression profiles of these small RNAs, which allowed us to predict new insights on their involvement in the biology of B. glabrata.

\section{MATERIALS AND METHODS}

Biological samples and RNA preparation - B. glabrata mollusks (Belo Horizonte strain - 056/2012/SECEX/CGEN) were obtained from Moluscario Lobato Paraense at the René Rachou Institute. For small RNA sequencing, 10 snails with 12 to $15 \mathrm{~mm}$ in diameter, were pooled for each group. The groups, with shell and without shell, were chosen to represent, respectively, mollusks with whole hemolymph and with poor amount of hemolymph. For quantitative polymerase chain reaction (qPCR), egg masses and snails at different developmental stages $(10,20$, and 40 days) were used. RNA preparations were made from triplicates of 10 snails per specific stage. Total RNA was isolated and used for sequencing and qPCR assays. The samples were frozen in liquid nitrogen, macerated and homogenised with Tri Reagent ${ }^{\circledR}$ (Sigma-Aldrich). The total RNA was finally treated with DNase (TURBO DNA-free kit Ambion ${ }^{\circledR}$ ), as recommended by the manufacturer and quantified by using Nano Drop nano-spectrometer. The RNA quality was analysed through capillary electrophoresis on Bioanalyzer $\left(\right.$ Agilent $\left.{ }^{\mathbb{R}}\right)$. cDNA synthesis was determined by High-capacity Kit (Life Technologies ${ }^{\mathrm{TM}}$ ) according to manufacturer's instructions.

Small RNA sequencing and quantitative reverse transcription polymerase chain reaction (RT-qPCR) The small RNA fraction was purified from total RNA of B. glabrata and used afterward for library construction and sequencing through Illumina HiSeq ${ }^{\mathrm{TM}} 2500$ system according to manufacturer's instructions. The sequences are available in the SRA database (PRJNA596001). For qPCR, TaqMan ${ }^{\circledR}$ system (Life Technologies ${ }^{\mathrm{TM}}$ ) was chosen and the assays were performed on ViiA $7^{\mathrm{TM}}$ Real-Time PCR System (Applied Biosystems ${ }^{\mathrm{TM}}$ ). The TaqMan ${ }^{\circledR}$ assay oligonucleotides were manufactured by Life Technologies ${ }^{\mathrm{TM}}$ [Supplementary data (Table I)]. The miRNAs expression level was measured in three biological replicates of snail samples from each different age and, gene expression levels were normalised by using the U6 transcript as internal control. The relative quantification of the expressed gene was determined by the $2^{-\Delta \Delta \mathrm{Ct}} \operatorname{method}^{(20)}$ and statistical analyses among the different groups was performed by using the One-Way ANOVA, with Tukey test as post-hoc test on GraphPad Prism $^{\circledR} 5.0$ (GraphPad Software, Inc., San Diego, USA). Values of $p \leq 0.05$ were considered statistically significant and are denoted with asterisks in the figures.

Small RNA sequencing data - The reads sequenced from the different samples of B. glabrata adult snails, with and without shell, were filtered according to the quality scores by using the FastQC software and the adapt- 
ers sequences trimmed through Trimmomatic software (http://www.usadellab.org/cms/?page=trimmomatic). Reads with length ranging from 15 to 35 nucleotides were selected and, those considered with low quality (quality score $<25$ ) were discarded from the study.

miRNA and piRNA analyses - All valid read sequences were counted and aligned onto B. glabrata genome (https:// www.vectorbase.org/organisms/biomphalaria-glabrata) by using bowtie andmiRDeep2 software (https://github. com/rajewsky-lab/mirdeep2). Precursor miRNA sequences used as reference for the analyses were obtained from Adema, 2017.(6) Beside this, the mature sequences used in the NGS analysis were also predicted from the alignment between the putative B. glabrata precursor miRNAs and their respective ortholog from animal species retrieved from miRBase database (http://www.miRbase.org/), accepting until five mismatches.

For the identification of miRNA target genes, we collected 3'-UTR-sequences from the annotation features defined in the GFF3 file from B. glabrata genome. Furthermore, in order to search for the gene targets, miRanda software (http://www.microrna.org/) and RNAhybrid software (http://bibiserv.techfak.uni-bielefeld.de/rnahybrid) were used. The miRanda software was set with the following parameters and conditions: a gap opening penalty of -8 , a gap extension penalty of -2 ; match with minimum score threshold 120 , target duplex with maximum threshold free energy $-15 \mathrm{kcal} / \mathrm{mol}$, scaling parameter three for complementary nucleotide match score, counting from the miRNA $5^{\prime}$ end, and demand strict 5 ' seed pairing between two and nine nucleotides. The RNAhybrid software was also used for the same purpose and it was set with default parameters. Only the miRNA target genes found in both software were considered for further analysis.
For the piRNA analysis, only reads with length between 23 and 35 nucleotides were included. Reads mapped onto Bgl-miRNAs and Bgl-rRNA sequences were filtered and removed from the study in this step. Predicted clusters of Bgl-piRNAs were obtained by using proTRAC software set according to the pipeline suggested by Rosenkranz. The ping-pong cycle signature was predicted by using PingPongPro software (https:// sourceforge.net/p/pingpongpro/), properly set to a predefined length of putative Bgl-piRNAs.

\section{RESULTS}

The small RNA sequencing for both samples, with and without shell, showed a large amount of reads with great diversity and quantity. The counts of unique and total reads suggested this diversity (Table I) with large differences for both samples. Sequences of 15 to $35 \mathrm{nu}-$ cleotides were kept for small RNAs analysis and over 43 million reads were obtained from samples without shell, while more than 26 million from samples with shell. The two groups showed similar distribution for read sizes, with typical length of the miRNAs sequences, ranging from 21 to 24 nucleotides and for piRNAs from 27 to 30 nucleotides (Fig. 1).

Initially, the raw data was compared with sequences on Rfam database, which allowed identification of small RNAs [Supplementary data (Fig. 1)] presented in the samples. Sixty eight pre-miRNAs annotated in the $B$. glabrata genome [Supplementary data (Table II)] were identified as precursors ${ }^{(6)}$ for 89 mature miRNAs identified in the small RNA sequencing samples (Table II).

The mature Bgl-miRNAs identified in small RNA sequenced libraries revealed a set of five Mollusk-specific miRNAs [Supplementary data (Fig. 2)]. In addition, some miRNAs stood out by presenting large reads counts,

TABLE I

Small RNA sequencing counts of reads

\begin{tabular}{|c|c|c|c|c|}
\hline & \multicolumn{2}{|c|}{ Without shell } & \multicolumn{2}{|c|}{ With shell } \\
\hline & Unique & Total & Unique & Total \\
\hline Reads (Adapter trimmed) & 1123762 & 44103293 & 2785427 & 26488178 \\
\hline Clean reads (15-35 nt) & 968931 & 43290179 & 2728664 & 26404729 \\
\hline Not matching genome & 624307 & 12344582 & 1759485 & 7598633 \\
\hline Matching genome & 344624 & 30945597 & 969179 & 18806096 \\
\hline Matching mature core miRNAs & 86 & 12629502 & 89 & 10236064 \\
\hline Matching piRNAs & 33377 & 2696565 & 209957 & 3954541 \\
\hline Matching Rfam database & 210527 & 17032569 & 251260 & 1938236 \\
\hline tRNA & 117015 & 15635721 & 87557 & 972458 \\
\hline rRNA & 73865 & 1192639 & 124043 & 718291 \\
\hline snoRNA & 1068 & 5195 & 2394 & 22094 \\
\hline snRNA & 762 & 22015 & 960 & 35491 \\
\hline other & 17817 & 176999 & 36306 & 189902 \\
\hline Unknown reads & 879772 & 11744657 & 2324121 & 10359337 \\
\hline
\end{tabular}




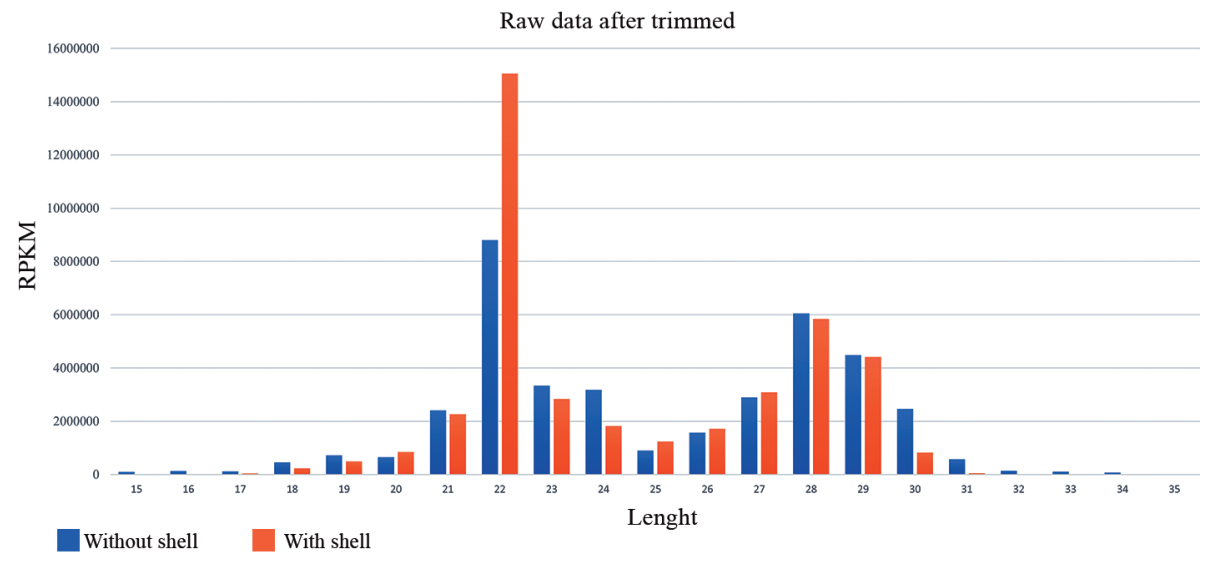

Fig. 1: raw data after trimmed. After trimming, reads sequences from Biomphalaria glabrata with and without shell revealed size distribution ranging from 21 to 24 and 27 to 30 suggesting a profile of miRNAs and piRNAs, respectively.

like bgl-bantam, bgl-let-7, bgl-miR-100, bgl-miR-184, bgl-miR-71, bgl-miR-1984, bgl-miR-1a, bgl-miR-315, bgl-miR-375, bgl-miR-750, bgl-miR-8 and bgl-miR-92. These mature Bgl-miRNAs represented $86 \%$ of total in the sample of snails without shell and $94 \%$ of those with shell (Fig. 2), suggesting these miRNAs may an important role in the snail biology and, for this, additional studies related to their putative targets were performed.

Identification of miRNA target genes in B. glabrata - The strategy of using two softwares for prediction and, accepting as valid only those genes predicted by both, allowed the identification of several target genes for all the mature Bgl-miRNAs [Supplementary data (Table III)]. In order to improve the power of prediction of the results and decrease false positives, mismatches between the seed region of the mature Bgl-miRNA sequences and their respective $3^{\prime}$ UTR target gene in $B$. glabrata genome were not accepted on both softwares. Over 40,000 predicted targets were identified by miRanda software and over 640,000 by RNAhybrid. However, only 26432 target genes were considered since they were identified by both softwares (Fig. 3A). Within these predicted targets, $73 \%$ represented known gene ontologies (GO) annotation, which supported the functional prediction of biological processes associated with miRNAs identified in this study (Fig. 3B).

$R T$-qPCR validation - After characterising B. glabrata miRNAs by high-throughput sequence method, the level of expression for the most outstanding miRNAs was measured by qPCR. A set of miRNAs including: bgl-miR-8, bgl-miR-92, bgl-miR-184, bgl-miR-315, bgl-miR-1984, bgl-miR-375 and bgl-miR-750 had their expression level evaluated in B. glabrata snails from different developmental stages by qPCR. Snails with 10, 20 and 40 days of age were selected as representative of different stages of the life cycle, including those sexually immature, in transition to adult stage and finally adult ones, ${ }^{(7)}$ Egg masses from B. glabrata were used as control.

There were significant differences in the miRNA expression in different stages of the snails $(p<0.05)$ (Fig. 4). This was more pronounced in the expression of bglmiR-184 (Fig. 4C) and bgl-miR-750 (Fig. 4G). In age 20 and 40 days snails, bgl-miR-184 was under-expressed. However, there was an over-expression of bgl-miR-750 in snails of all ages analysed. Our results also showed thatbgl-miR-8 (Fig. 4A) and bgl-miR-92 (Fig. 4B) were significantly expressed in snails age 10 and 20 days. Similar observation was recorded for bgl-miR-184 expression. The bgl-miR-1984 (Fig. 4E) showed significant differences in its expression in 10, 20 and 40 days. No significant difference in expression was observed for bgl-miR-315 (Fig. 4D) and bgl-miR-375 (Fig. 4F) relative to snails ages.

Identification of putative piRNAs on B. glabrata The piRNAs repertoire in B. glabrata showed a large count of reads with 28 to 29 nucleotides of length in both libraries of snails with and without shell [Supplementary data (Fig. 3)]. These sequences were compared, firstly, with Bgl-miRNA precursors and Bgl-rRNAs. Only reads unmapped onto those types of RNAs were considered for Bgl-piRNAs search.

Filtered sequences corresponding to putative BglpiRNAs were analysed with ProTrac software (https:// sourceforge.net/projects/protrac/) to predict piRNA clusters based on genome mapped piRNA sequence reads. The typical signatures of piRNAs, the presence of $1 \mathrm{U}$ or $10 \mathrm{~A}$ on sequence, were used as attribute to infer the existence of classical piRNAs. It was found that 195 predicted clusters in the library constructed with samples of snails without shell [Supplementary data (Table IV)] comprise 49,155 putative piRNAs. A total of 352 predicted clusters in the library of snails with shell, comprised 314,729 putative piRNAs [Supplementary data (Table V)]. The sequences of putative piRNAs for both libraries were concatenated [Supplementary data (Table VI)] and the result of putative Bgl-piRNAs was presented as expected abundance of sequences with 27 to 29 nucleotides in length (Fig. 5).

Beyond the typical signature 1U-10A (Fig. 6), which is accordance with what is defined in other organisms as the ping pong cycle signature was also found in both libraries of snails with and without shell, further high scores for A at 10th position [Supplementary data (Fig. 4)]. Taken together, these findings reinforce the identification of piRNAs in B. glabrata genome. 
TABLE II

Characterisation of mature miRNAs on Biomphalaria glabrata

\begin{tabular}{|c|c|c|c|c|c|c|c|}
\hline \multirow[b]{2}{*}{ Mature miRNA name } & \multirow[b]{2}{*}{ Mature sequence } & \multirow{2}{*}{ 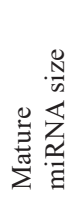 } & \multirow{2}{*}{ 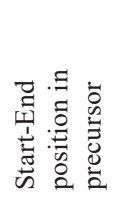 } & \multirow{2}{*}{ 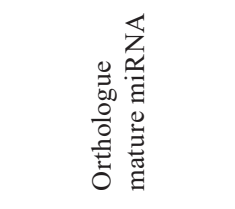 } & \multirow{2}{*}{ 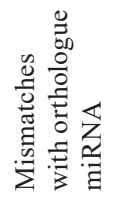 } & \multicolumn{2}{|c|}{ Number of reads in NGS } \\
\hline & & & & & & Without shell & With shell \\
\hline bgl-bantam-3p & ugagaucauugugaaaacugauu & 23 & $47-69$ & ame-bantam & 1 & 113854 & 1307 \\
\hline bgl-bantam-5p & cugguuuucccauuggucuggcag & 24 & $10-33$ & tca-bantam-5p & 5 & 8 & 97 \\
\hline bgl-let-7-5p & ugagguaguagguuguauuguu & 22 & $2-23$ & cel-let-7-5p & 1 & 156393 & 2006275 \\
\hline bgl-miR-100-3p & acaaguuugcaucuauggguaug & 23 & $58-80$ & tgu-miR-100-3p & 4 & 27 & 13 \\
\hline bgl-miR-100-5p & aacccguagaaccgaacuugugc & 23 & $17-39$ & oha-miR-100-5p & 1 & 4489426 & 12675 \\
\hline bgl-miR-1175-3p & ugagauucaacuccuccaacugc & 23 & $59-81$ & cte-miR-1175-3p & 0 & 8517 & 769 \\
\hline bgl-miR-1175-5p & aguggagagaguuuuaucucau & 22 & $18-39$ & cte-miR-1175-5p & 0 & 1037 & 921 \\
\hline bgl-miR-124-3p & uaaggcacgcggugaaugccaag & 23 & $57-79$ & dme-miR-124-3p & 0 & 347 & 2440 \\
\hline bgl-miR-124-5p & cgcguucacugggucagccuug & 22 & $20-41$ & tur-miR-124-1-5p & 5 & 0 & 1 \\
\hline bgl-miR-125-5p & ucccugagaccauaauuugug & 21 & $20-40$ & dme-miR-125-5p & 2 & 17076 & 57332 \\
\hline bgl-miR-12-5p & ugaguauuacuucagguacug & 21 & $10-30$ & bmo-miR-12 & 0 & 81532 & 2164 \\
\hline bgl-miR-1260-3p & aucccaccgcugccacca & 18 & $50-67$ & cgr-miR-1260 & 0 & 13 & 273 \\
\hline bgl-miR-133-3p & uuugguccccuucaaucaguugua & 24 & $57-80$ & efu-miR-133-3p & 2 & 6334 & 24934 \\
\hline bgl-miR-133-5p & agcugguugaaucugggccaaau & 23 & $17-39$ & lgi-miR-133-5p & 2 & 1916 & 13 \\
\hline bgl-miR-137-3p & uuauugcuugagaauacacgua & 22 & $52-73$ & ame-miR-137 & 0 & 147 & 179 \\
\hline bgl-miR-137-5p & acggguauucuuggguaaauaau & 23 & $15-37$ & oha-miR-137-5p & 1 & 0 & 9 \\
\hline bgl-miR-153-3p & uugcauagucacaaaagugauc & 22 & $59-80$ & mmu-miR-153-3p & 0 & 1970 & 70 \\
\hline bgl-miR-184-3p & acuggacggagaacugauaagggc & 24 & $72-95$ & bmo-miR-184-3p & 0 & 2185751 & 2402352 \\
\hline bgl-miR-190-5p & agauauguuugauauauuuggugg & 24 & $18-41$ & cte-miR-190 & 0 & 3534 & 584 \\
\hline bgl-miR-193-3p & uacuggccuucaaaaucccaa & 21 & $52-72$ & lgi-miR-193 & 1 & 1137 & 3766 \\
\hline bgl-miR-1984-5p & ugcccuauccgucaggaacugug & 23 & $9-31$ & hru-miR-1984 & 0 & 403721 & 144101 \\
\hline bgl-miR-1985-5p & ugccauuuuuaucagucacugug & 23 & $17-39$ & hru-miR-1985 & 0 & 127450 & 14703 \\
\hline bgl-miR-1986-3p & uggauuucccaagauccgugau & 22 & $57-68$ & hru-miR-1986 & 0 & 40 & 7522 \\
\hline bgl-miR-1990-3p & cgggacuacgucaacu & 16 & $56-71$ & cte-miR-1990c-3p & 2 & 637 & 1228 \\
\hline bgl-miR-1990-5p & aguaaguugauggggucccagg & 22 & $20-41$ & hru-miR-1990 & 0 & 144 & 392 \\
\hline bgl-miR-1991-5p & cuuacccuguuauacugagaagu & 23 & $16-38$ & hru-miR-1991 & 3 & 541 & 114 \\
\hline bgl-miR-1992-3p & ucagcaguuguaccacugauuug & 23 & $59-81$ & lgi-miR-1992 & 0 & 180 & 1255 \\
\hline bgl-miR-1993-3p & uauuaugcugcuauucacgaga & 22 & $45-66$ & cte-miR-1993 & 1 & 432 & 160 \\
\hline bgl-miR-1994a-3p & ugagacaguguguccucccuug & 22 & $53-74$ & lgi-miR-1994a & 0 & 1053 & 779 \\
\hline bgl-miR-1994b-3p & ugagacagugcguccucccuca & 22 & $51-72$ & lgi-miR-1994b & 1 & 945 & 4911 \\
\hline bgl-miR-199a-3p & gcaguagucugcacauuguuua & 22 & $62-83$ & mmu-miR-199a-3p & 2 & 0 & 1 \\
\hline bgl-miR-1a-3p & uggaauguaaagaaguauguau & 22 & $69-90$ & mmu-miR-1a-3p & 0 & 978 & 3867163 \\
\hline Bgl-miR-1a-5p & acauacuucuuugcuaucccau & 22 & $32-53$ & mmu-miR-1a-5p & 4 & 7 & 572 \\
\hline bgl-miR-2001-5p & uugugaccguuauaaugggcauu & 23 & $20-42$ & lgi-miR-2001 & 0 & 11799 & 2051 \\
\hline bgl-miR-216a-5p & uaaucucagcugguaauucagag & 23 & $10-32$ & lgi-miR-216a & 1 & 3141 & 123 \\
\hline bgl-miR-216b-5p & uaauaucagcugguaauccugag & 23 & $9-31$ & lgi-miR-216b & 0 & 25179 & 804 \\
\hline bgl-miR-219a-3p & agaacuguguguggacaucagu & 22 & $50-69$ & ipu-miR-219a & 4 & 46 & 41 \\
\hline bgl-miR-219a-5p & ugauuguccaaacgcaauucuug & 23 & $22-44$ & ssa-miR-219a-5p & 0 & 51 & 269 \\
\hline bgl-miR-252a-5p & cuaaguacuggugecgcggga & 21 & $24-44$ & lgi-miR-252a & 0 & 16404 & 40203 \\
\hline bgl-miR-252b-3p & accugcacaccggugcuua & 19 & $55-73$ & str-miR-252b-3p & 3 & 4 & 1 \\
\hline bgl-miR-252b-5p & auaaguaguggugccgcaggua & 22 & $18-39$ & lgi-miR-252b & 0 & 986 & 35027 \\
\hline bgl-miR-2722-3p & uggcgccguggaaacaucuacc & 22 & $51-72$ & lgi-miR-2722 & 0 & 685 & 132 \\
\hline bgl-miR-277a-3p & uaaaugcauuaucugguaucu & 21 & $60-80$ & cte-miR-277a & 1 & 79902 & 7332 \\
\hline bgl-miR-278-3p & ucggugggacuuucguucguuu & 22 & $62-83$ & sko-miR-278 & 0 & 25242 & 102 \\
\hline bgl-miR-279-3p & ugacuagauccacacucaucca & 22 & $55-76$ & lgi-miR-279 & 0 & 50935 & 35566 \\
\hline bgl-miR-279-5p & gauggcuguuggucuggugcaug & 23 & $19-41$ & tca-miR-279b-5p & 4 & 488 & 1168 \\
\hline bgl-miR-281-3p & acugucauggaguugcucucuu & 22 & $42-63$ & bmo-miR-281-3p & 0 & 4685 & 39 \\
\hline bgl-miR-281-5p & aagggagcauccgucgacagu & 21 & $9-29$ & lgi-miR-281-5p & 1 & 126826 & 4808 \\
\hline bgl-miR-29a-3p & uagcaccauuugaaaucaguuu & 22 & $62-83$ & cte-miR-29a & 0 & 553 & 3206 \\
\hline bgl-miR-29b-3p & uagcaccauuugaaaucaguuu & 23 & $55-77$ & bfl-miR-29b-3p & 0 & 553 & 3206 \\
\hline bgl-miR-2a-1-3p & uaucacagccagcuuugaugagcg & 24 & $54-77$ & api-miR-2a & 0 & 7195 & 11517 \\
\hline
\end{tabular}




\begin{tabular}{|c|c|c|c|c|c|c|c|}
\hline \multirow[b]{2}{*}{ Mature miRNA name } & \multirow[b]{2}{*}{ Mature sequence } & \multirow{2}{*}{ 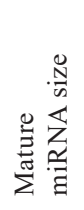 } & \multirow{2}{*}{ 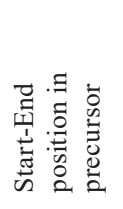 } & \multirow{2}{*}{ 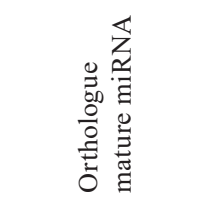 } & \multirow{2}{*}{ 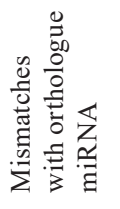 } & \multicolumn{2}{|c|}{ Number of reads in NGS } \\
\hline & & & & & & Without shell & With shell \\
\hline bgl-miR-2a-2-3p & uaucacagccagcuuugauga & 21 & $52-72$ & dme-miR-2a-3p & 0 & 15098 & 46019 \\
\hline bgl-miR-2a-2-5p & cgucaaggcgguugugaugug & 21 & $16-36$ & asu-miR-2a-5p & 4 & 206 & 577 \\
\hline bgl-miR-2b-3p & uaucacagucagcuuugaugagcu & 24 & $57-81$ & aae-miR-2b & 1 & 15098 & 13687 \\
\hline bgl-miR-2d-3p & uaucacagccugcuuggaucagu & 23 & $53-75$ & lgi-miR-2d & 0 & 14506 & 4997 \\
\hline bgl-miR-315-5p & uuuugauuguugcucagaaagcc & 23 & $11-33$ & cte-miR-315 & 0 & 1607001 & 30357 \\
\hline bgl-miR-317-3p & ugaacacagcuggugguaucuuau & 24 & $56-79$ & lgi-miR-317 & 1 & 46000 & 14531 \\
\hline bgl-miR-33-3p & caaugucucugcagugcaau & 20 & $52-71$ & oan-miR-33a-3p & 2 & 160 & 18 \\
\hline bgl-miR-33-5p & gugcauuguaguugcauugcgug & 23 & $18-40$ & tca-miR-33-5p & 1 & 218 & 808 \\
\hline bgl-miR-34-3p & caaccacucuccacauuaccgcc & 23 & $51-73$ & aae-miR-34-3p & 5 & 54 & 29 \\
\hline bgl-miR-34-5p & uggcagugugguuagcugguugu & 23 & $17-39$ & dme-miR-34-5p & 0 & 7617 & 21912 \\
\hline bgl-miR-36a-3p & ucaccggguauacauucauccg & 20 & $56-75$ & asu-miR-36a-3p & 0 & 2 & 5 \\
\hline bgl-miR-375-3p & uuuguucguucggcucgcguuau & 23 & $61-83$ & bbe-miR-375-3p & 0 & 433042 & 774752 \\
\hline bgl-miR-67-1-5p & ucacaaccugcuugaaugaggac & 23 & $23-45$ & lgi-miR-67 & 0 & 731134 & 52870 \\
\hline bgl-miR-67-2-3p & ucacaaccugcuugaaugaggac & 23 & $50-72$ & lgi-miR-67 & 1 & 731134 & 52870 \\
\hline bgl-miR-71-5p & ugaaagacauggguagugagaug & 23 & $18-40$ & cte-miR-71 & 0 & 10395 & 337118 \\
\hline bgl-miR-72-3p & agcugugucauauguugcca & 20 & $52-71$ & str-miR-72-3p & 4 & 19 & 75 \\
\hline bgl-miR-72-5p & aggcaagauguuggcauagcuga & 23 & $25-37$ & cel-miR-72-5p & 0 & 11838 & 15097 \\
\hline bgl-miR-7-3p & caauaaaucacaaucuuc & 18 & $57-74$ & bbe-miR-7-3p & 2 & 61 & 6 \\
\hline bgl-miR-745a-3p & agcugccugaugaagagcugu & 21 & $55-75$ & lgi-miR-745a & 0 & 23721 & 24336 \\
\hline bgl-miR-745b-3p & agcugccaaaugaagggcugu & 21 & $49-69$ & lgi-miR-745b & 0 & 64818 & 2126 \\
\hline bgl-miR-750-3p & ccagaucuaacucuuccagcuca & 23 & $60-82$ & cte-miR-750 & 0 & 534947 & 9893 \\
\hline bgl-miR-750-5p & cguuggaggauuggaucuuagc & 22 & $22-43$ & tca-miR-750-5p & 5 & 2354 & 25 \\
\hline bgl-miR-7-5p & uggaagacuagugauuuaguuguu & 24 & $18-41$ & ssc-miR-7 & 1 & 9 & 144 \\
\hline bgl-miR-8-3p & uaauacugucagguaaagauguc & 23 & $63-85$ & dme-miR-8-3p & 0 & 245404 & 47380 \\
\hline bgl-miR-8-5p & cgucuuaccuagcagcauugga & 22 & $18-39$ & dvi-miR-8-5p & 4 & 528 & 181 \\
\hline bgl-miR-87b-1-3p & gugagcaaaguuucagguguau & 22 & $60-81$ & cte-miR-87b & 0 & 2967 & 10380 \\
\hline bgl-miR-87b-2-3p & gugagcaaaguuucagguguau & 22 & $59-80$ & cte-miR-87b & 0 & 2967 & 10380 \\
\hline bgl-miR-92-3p & aauugcacucgucceggccugc & 22 & $60-81$ & dpu-miR-92 & 0 & 651682 & 30648 \\
\hline bgl-miR-92a-1-3p & uauugcacuuuucceggccugu & 22 & $57-78$ & hsa-miR-92a-3p & 2 & 57848 & 11249 \\
\hline bgl-miR-92a-3p & uauugcacuuuuccaggccuuu & 22 & $56-77$ & bfl-miR-92a & 2 & 1048 & 2086 \\
\hline bgl-miR-92b-3p & aauugcacuaaucccggccuac & 22 & $54-75$ & dme-miR-92b-3p & 2 & 16497 & 41985 \\
\hline bgl-miR-96a-5p & cuuggcacuggcggaauaguca & 22 & $10-31$ & lgi-miR-96a & 1 & 5745 & 25313 \\
\hline bgl-miR-96b-5p & auuuggcacuuguggaauaaucg & 23 & $16-38$ & lgi-miR-96b & 0 & 7132 & 729 \\
\hline bgl-miR-981-3p & uucguugucgucgaaaccugccu & 23 & $50-72$ & cte-miR-981 & 1 & 153740 & 15044 \\
\hline bgl-miR-9a-3p & auaaagcuagguuaccaaaggc & 23 & $47-68$ & lgi-miR-9-3p & 0 & 990 & 945 \\
\hline bgl-miR-9a-5p & ucuuugguuaucuagcuguauga & 22 & $9-31$ & dme-miR-9a-5p & 0 & 18353 & 817 \\
\hline bgl-miR-9b-3p & auaaagcuagguuaccaaaggc & 22 & $47-68$ & lgi-miR-9-3p & 0 & 990 & 945 \\
\hline bgl-miR-9b-5p & cuuugguaaccuagcuuuauga & 22 & $1-23$ & ame-miR-9b & 2 & 8 & 16 \\
\hline
\end{tabular}

\section{DISCUSSION}

The susceptibility of B. glabrata to $S$. mansoni infection has been studied by different research groups around the world, some of whom attempted to unravel the genetic or physiologic mechanisms that regulate $B$. glabrata susceptibility to $S$. mansoni nfection. However, no study is currently available that elucidates the role of small RNAs in the snail host-parasite relationship. Our group has previously studied the expression profile of genes on miRNA biogenesis in the mollusk. ${ }^{(7)}$ In present the study, we were able to reveal numerous small RNAs expressed in different developmental stages of B. gla- brata using a small RNA high throughput sequencing method and bioinformatics tools, thus predicting their involvement in regulating essential processes in the snail biology. The results presented in our study open the way to exploration of roles of small RNAs in B. glabrata development and susceptibility to infection by $S$. mansoni.

In this study, we used samples of the snails with and without shell. Snails without shell lose almost the whole amount of hemolymph during the process of shell removal. On the other hand, the samples comprising snails with shell keep their complete molecular contents. By using the two types of snails we could show differences between their repertoires of small RNAs, which sug- 


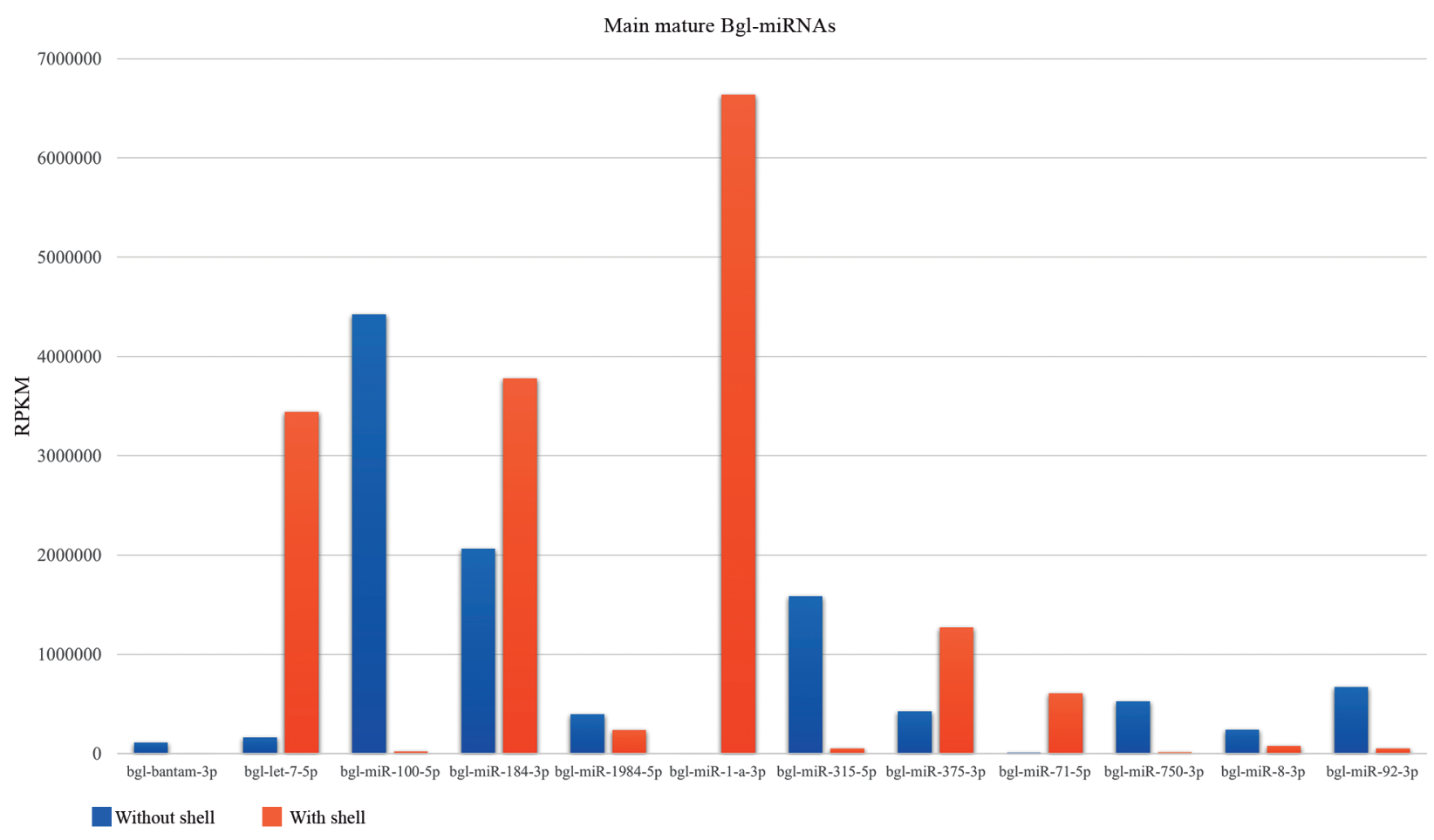

Fig. 2: the most abundant Bgl-miRNAs in the samples sequenced. Mature miRNAs with larger counts of mapped reads in snails with shell and without shell.

gested the importance of the hemolymph in the natural diversity of these molecules in B. glabrata. Previous studies have shown the importance of hemolymph as an essential factor to snail in fighting infection. . $^{(3,4,21)}$ The additional small RNAs observed in sample with preserved hemolymph are predicted to play additional functional roles in snail protection against Schistosoma infection.

When filtered to the range of 15 to 35 nucleotides, the sequenced reads with high quality revealed that the libraries presented a similar outline of small RNA lengths (Fig. 1). The reads found with 21 to 24 nucleotides in length are compatible with typical miRNAs mean size. These sequences were aligned onto the Rfam database [Supplementary data (Fig. 1)] and the highest counts contributed to enrich the miRNAs like when compared to piRNAs. Otherwise, the distribution of the reads between 27 to 30 nucleotides was compatible with piRNA sequences and, instead of the smaller counts per each sequence, a larger diversity of Bgl-piRNAs was found when compared to miRNAs and fragments of tRNA. The results of Bgl-piRNAs showed a larger diversity of sequences in snails with shell compared to those without shell, suggesting that the hemolymph of the B. glabrata has an expressive amount of circulating piRNAs. It is worth highlighting that small RNAs, especially piRNAs, are remarkably present in human blood, suggesting their importance as biomarkers for proliferative disorders. ${ }^{(22)}$

The results also suggested a wide repertoire of BglmiRNAs by searching for mature and precursor sequences of miRNAs on the B. glabrata genome [Supplementary data (Table II)] as previously evidenced.
(6) We identified 89 mature Bgl-miRNAs related to 68 Bgl-miRNA precursors (Table II), some of which are conserved in metazoan, such as bgl-bantam, bgl-let-7, bgl-miR-100, bgl-miR-184, bgl-miR-71, bgl-miR-1984, bgl-miR-1a, bgl-miR-315, bgl-miR-375, bgl-miR-750, bgl-miR-8 and bgl-miR-92.

The bgl-miR-100 had the highest number of reads aligned (4 million reads) onto the without shell snail library, while bgl-miR-1a had more than 2 million reads aligned against both snail libraries (with and without shell). The miR-100 seems to be involved in controlling cell growth, ${ }^{(23)}$ as well as in the immune system activity. ${ }^{(24)}$ The large abundance of bgl-miR-100 is even more significant since its analogous miR-100 takes part in the regulation of metabolism and longevity of the Drosophila melanogaster. ${ }^{(25)}$ In $B$. glabrata, the bgl-miR-100 has E3 ubiquitin ligase as a target, and other genes involved in cell biology regulation ${ }^{(26)}$ and lipids and sugar metabolism [Supplementary data (Table III)].

The bgl-miR-1a also seems to be related to cell growth regulation; acting as a tumor suppressor ${ }^{(27)}$ and regulating heart development in mammalians. ${ }^{(28)}$ It may affect the development of somatic muscle cells by interfering with E3 ubiquitin ligase activity. ${ }^{(29)}$ The targets prediction on B. glabrata 3' UTR showed that bgl-miR-la can regulate ubiquitin proteins, and targets related to muscular organisation, such as actin and fibril in, corroborating with findings in literature [Supplementary data (Table III)]. It is also interesting to have observed a significant number of predicted targets on B. glabrata are involved in response to stress, which may be determinants of the 


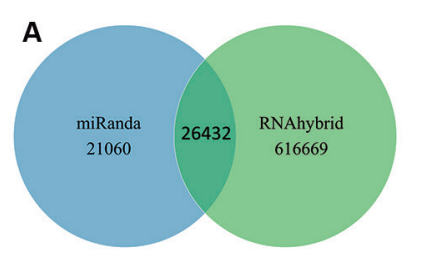

B Bgl-miRNAs targets prediction

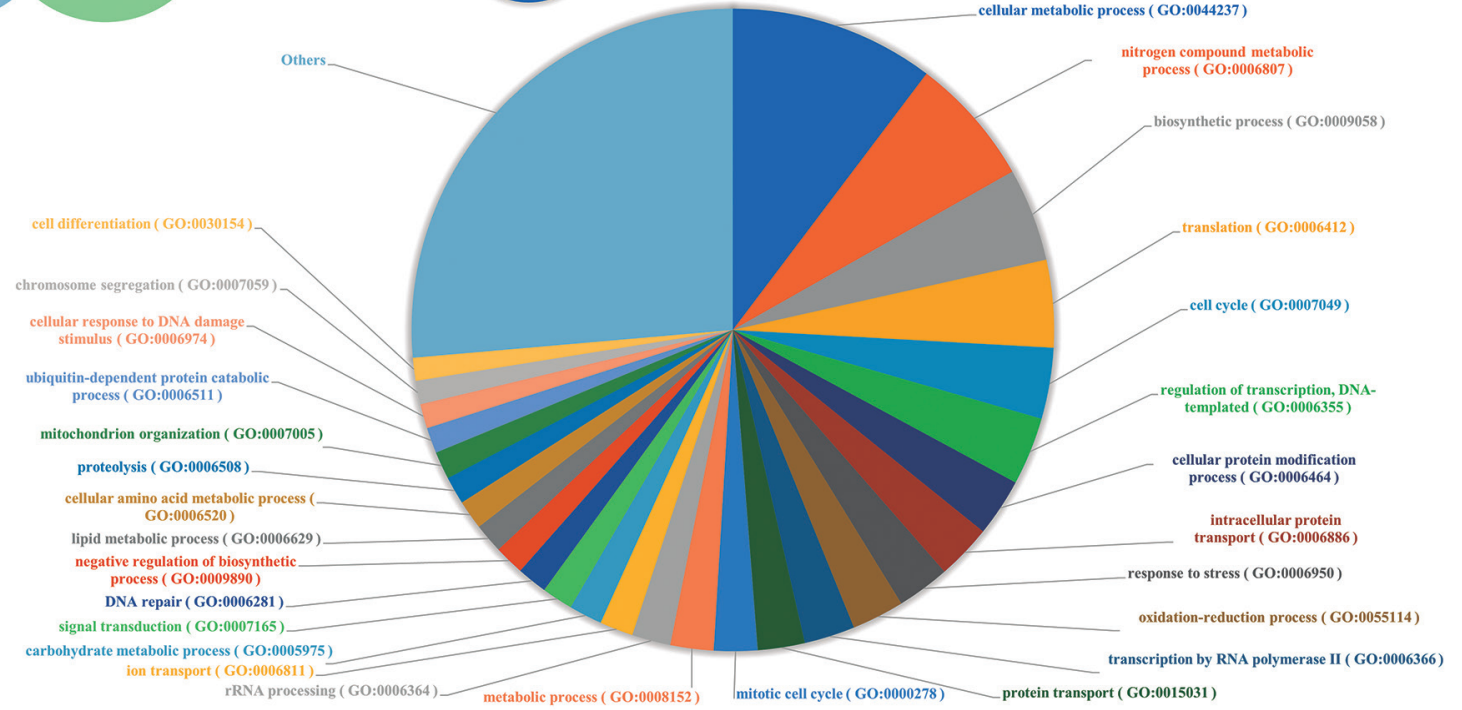

Fig. 3: prediction of target-genes for Bgl-miRNAs in Biomphalaria glabrata. (A) 47492 putative targets found with miRanda software on B. glabrata 3'UTR se quences of genes, and 643101 with RNAhybrid. Only 26432 are common between them; (B) for the common predicted targets, $73 \%$ showed known GO. Those pertaining to the level of biological process were used to group the gene targets of Bgl-miRNAs.

snail adaptive process to the constant colonisation by numerous microorganisms. In summary, the increased expression of bgl-miR-100 and bgl-miR-la evidenced in our study, may play an important role in regulating the development of $B$. glabrata and response to infection.

The remaining miRNAs identified did not show such meaningful abundance, however, they stand out by their probable importance to B. glabrata based on their recognised functions in the biology of other organisms as seen in bantam. This miRNA has been reported to play a role in controlling the brain cell growth and feedback regulation of the Notch signaling pathway. ${ }^{(30)}$ It is suggested the bgl-bantam acts on the development of neuronal cells, mainly in those of tentacle ganglion, a region with dense olfactory neuropils distribution, where there is regular contact of snails with the environment. ${ }^{(31)}$ In $B$. glabrata, bantam showed a huge difference between the abundance of aligned reads in snail samples without shell $(113,854)$ and with shell $(1,307)$. The targets for this miRNA in $B$. glabrata suggest it can act in regulating the function of the neuronal cell by silencing synapse associated genes. We further suggest a function for bglbantam in the regulation of snail metabolism, since it recognises targets with redox function, also with regulatory action over the ubiquitin-protein complex [Supplementary data (Table III)].

The mature bgl-miR-71 presented 10,395 reads aligned onto the libraries of snail without shell against337,118 reads onto libraries of those with shell thus, suggesting its higher abundant in the hemolymph. Previous studies have suggested that, in Caenorhabditis elegans, miR-71 is involved in longevity by functioning mainly in the ner- vous system by helping in the maintenance of the proper responses to oxidative stress and thermal shock. ${ }^{(32)}$ In Marsupenaeus japonicus, this miRNA regulates the activation of apoptosis and downregulates phagocytosis, and the pro-phenoloxidase system, indicating its function in the innate immune system of invertebrates. ${ }^{(33)}$ In B. glabrata, the genes RAD50-like for DNA repair, E3 ubiquitin-protein ligase UBR3-like and toll-like receptor 3 [Supplementary data (Table III)] were predicted as targets tobgl-mir-71, suggesting it may be important in regulation of mollusk immune system.

The bgl-miR-184 has been linked to a different sort of cellular mechanisms in vertebrates and invertebrates organisms. In M. japonicus, this mature miRNA may regulate phagocytosis positively, ${ }^{(33)}$ similar to our results of target prediction do it. Moreover, in crustacean infected with the virus of white spot syndrome, miR184 was overexpressed when compared to non-infected one $^{(34)}$ suggesting its participation in organism immune defense. The bgl-miR-184 has high counts of reads aligned onto its mature sequence in both samples, which may reflex its importance for the mollusk. According to bgl-miR-184 target prediction, it is possible to predict it may act as a regulator of metabolic enzymes, such as aminoacylase-1A-like and ADP-ribosylation factor-like, and also act as a regulator of apoptosis and proteolysis processes [Supplementary data (Table III)], thus modulating aging, like in D. melanogaster. ${ }^{(25)}$

The let-7 miRNA is considered a potential regulator of development in C. elegans, starting its expression in the larval stage and extending to adulthood. ${ }^{(35)}$ In Anoph- 
eles stephensi, this microRNA is differentially expressed between males and females individuals. Furthermore, in D. melanogaster, it is significantly more expressed in pupae than in larvae, ${ }^{(36)}$ also suggesting its involvement in aging modulation and development of nervous tissue. (25) The counts of reads aligned to mature sequences of bgl-let-7 in the libraries of snail with shell were hugely higher (over 2 million reads) than those aligned onto the mature sequence of the libraries of snails without shell
(156,393 reads). This large difference reflects a higher availability of this miRNA in the hemolymph compared to snail tissues. According to the target prediction, bgllet-7 probably regulates lipid metabolism by controlling the expression of enzymes such as diacylglycerol lipase. It also shows that miRNA also regulates the proteolysis process mediated by ubiquitin by controlling the activity of enzymes such as ubiquitin carboxyl terminal hydrolase [Supplementary data (Table III)].
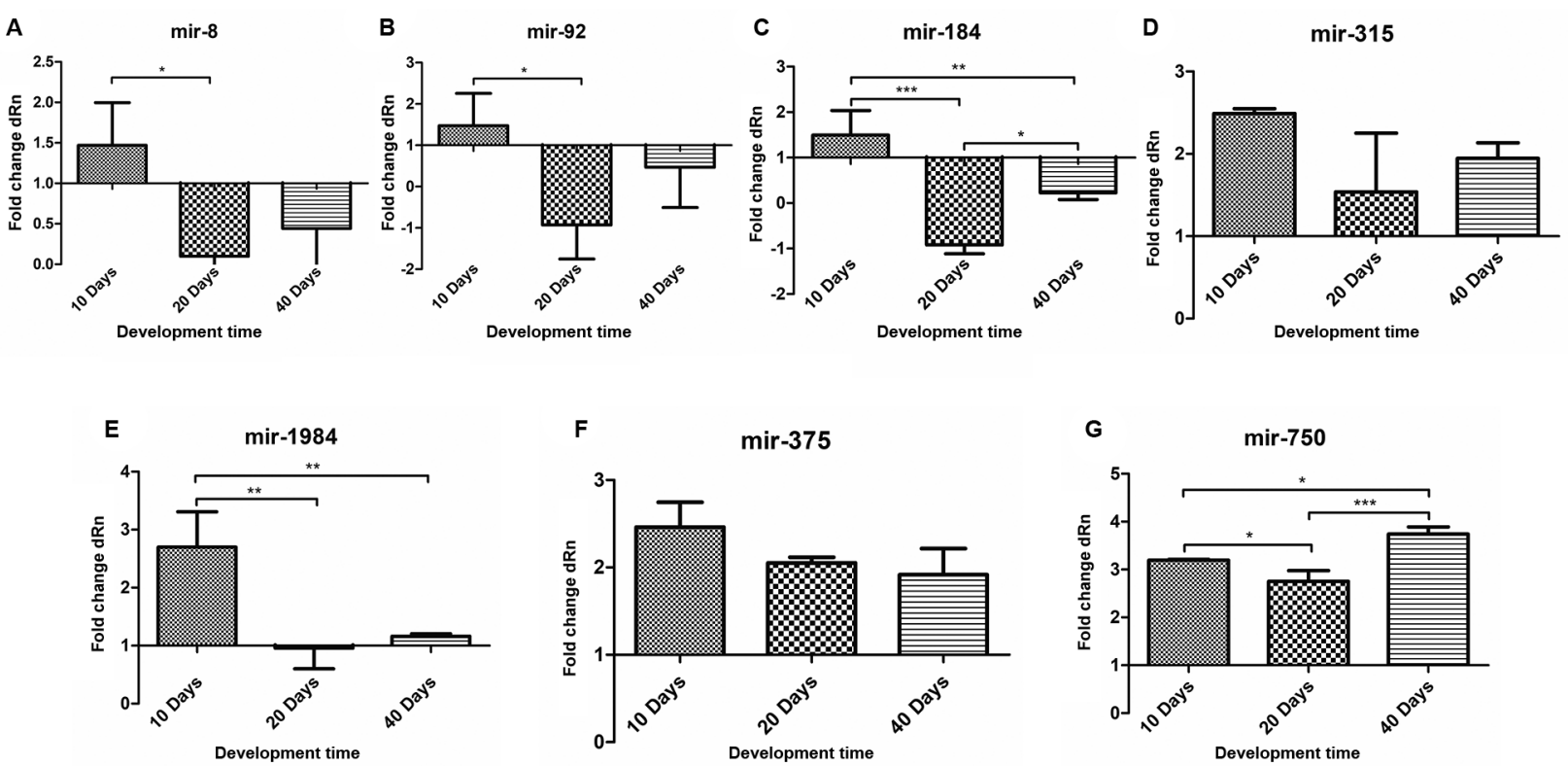

Fig. 4: Bgl-miRNAs expression determined through quantitative reverse transcription polymerase chain reaction (RT-qPCR) in developmental stages of Biomphalaria glabrata. (A) bgl-miR-8 showed significant difference between 10 and 20 days. (B) bgl-miR-92 showed significant difference between 10 and 20 days. (C) bgl-miR-184 showed significantly different profile between all evaluated time. (D) bgl-miR-315 not presented nonsignificant difference between evaluated time. (E) blg-miR-1984 showed a significant over-expression when compared with 20 and 40 days. (F) bgl-miR-375 not presented nonsignificant difference between evaluated time. (G) bgl-miR-750 showed profile significantly different between all evaluated time.

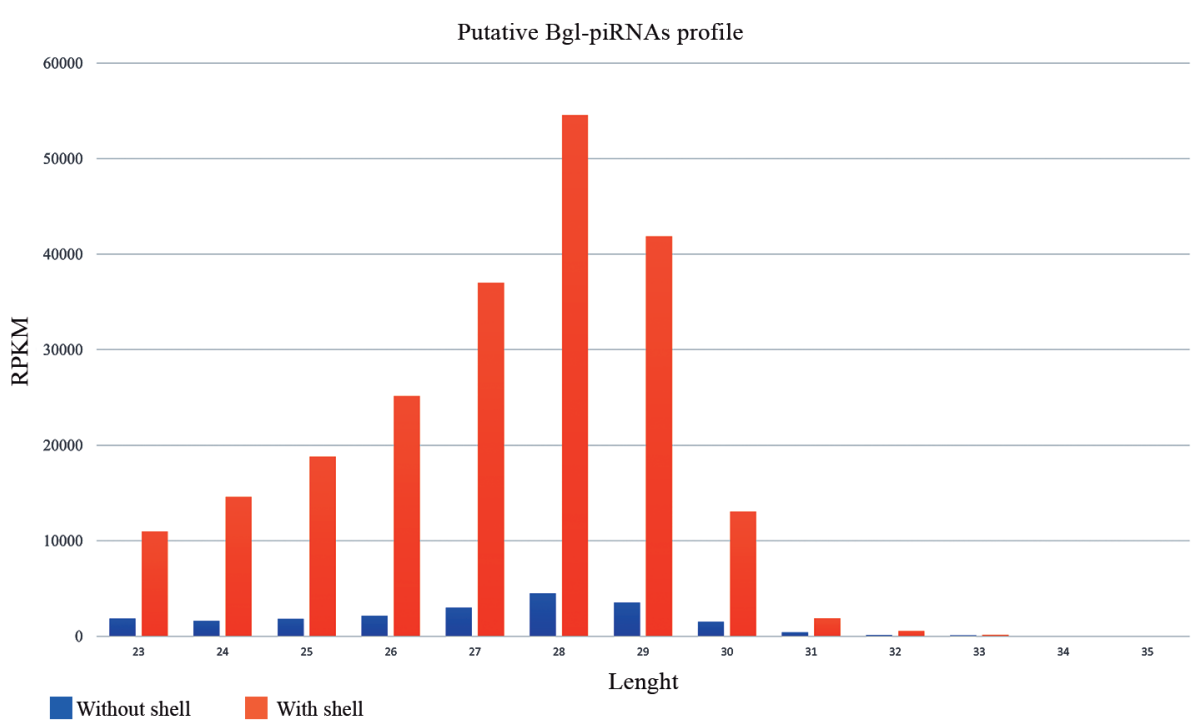

Fig. 5: putative piRNA sequences in Biomphalaria glabrata. Distribution of putative Bgl-piRNAs separated by size, demonstrating a larger diversity of piRNAs at library of snail with shell in relation to library of snail without shell. 

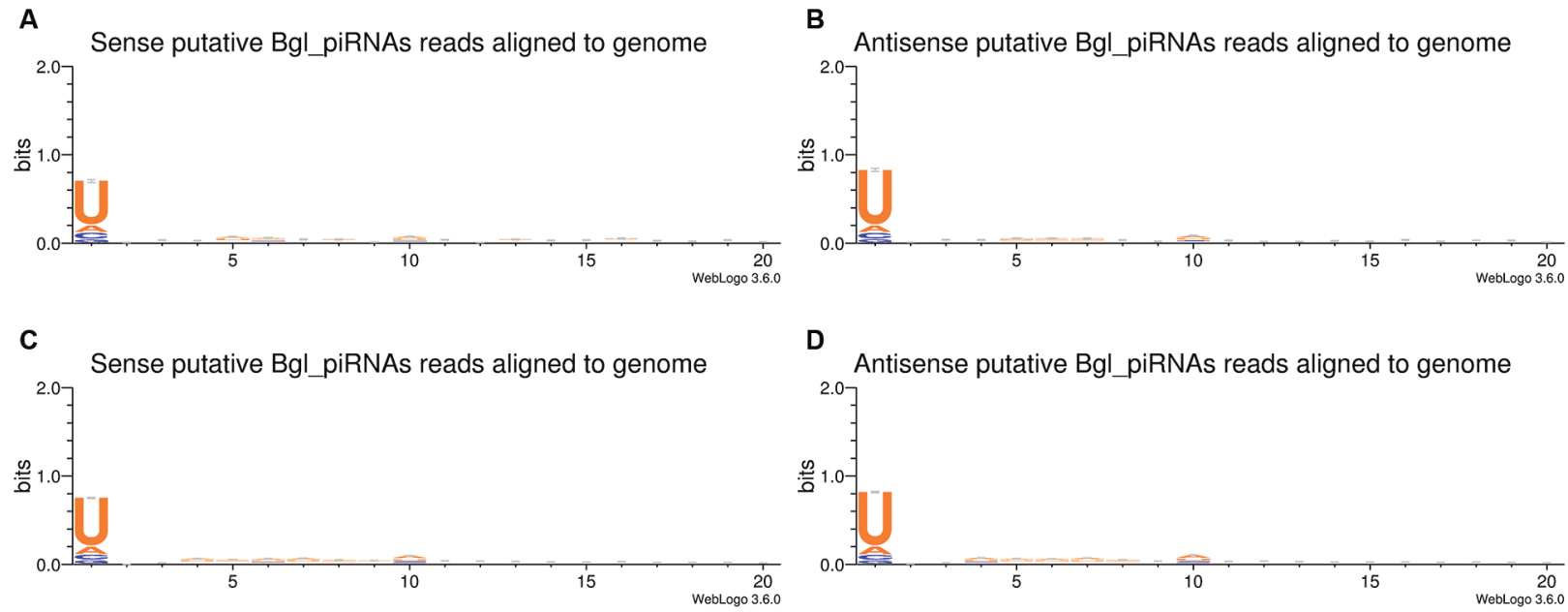

Fig. 6: sequence logo of putative Bgl-piRNAs sequences. The sequence logo was generated by using the putative Bgl-piRNA sequences trimmed of 1 to 20 nucleotides. (A) sequence logo of strand sense of the samples without shell with remarkable uridine at first position. (B) sequence logo of strand antisense of the samples without shell with remarkable uridine at first position. (C) sequence logo of strand sense of the samples with shell with remarkable uridine at first position. (D) sequence logo of strand antisense of the samples with shell with remarkable uridine at first position.

All the Bgl-miRNAs identified in the present study had more than one predicted gene target on the B. glabrata genome, similar to the well-recognised potential of miRNAs to act on several targets. ${ }^{(12)}$ The two softwares used for miRNA target prediction showed different possibilities, however, only the genes predicted by both were accepted as target for Bgl-miRNAs. Through the inspection of the annotations of predicted target genes, it is reasonable to infer that many biological processes are regulated by Bgl-miRNAs, including reproduction, growth, immune process, cell proliferation, metabolic process, and developmental process (Fig. 3B). These results confirmed that Bgl-miRNAs are probably involved in regulations of biological mechanisms such as development of the snail, as expected of this kind of biomolecule. Considering the fact that some genes which are strictly involved in snail immune response to infections were found as targets for Bgl-miRNAs sequenced, it is normal to predict that these molecules may act in regulating $B$. glabrata susceptibility to infection by $S$. mansoni. The involvement of the molecule in modulation of B. glabrata/S. mansoni relationship during infection will be investigated in upcoming studies.

Seven of the identified Bgl-miRNAs markedly conserved at the animal clades had their expression profiles further analysed through RT-qPCR. Their level of expression was assessed through different developmental life stages of B. glabrata: 10 days (young and sexual immature snails); 20 days (snails in transition to sexual maturity), and 40 days (snails in the adult stage, with complete sexual maturity). ${ }^{(7)}$ The gene expression in these three stages was compared to that in the pool of $B$. glabrata egg masses control. The precise degree of sexual maturity of the B. glabrata snail is poorly studied and not always consensual and, when explored, the authors prefer measuring the diameter of the shell to differentiate adults from young snails. In the present study, we chose a more precise and coherent approach, considering the counting of days of life as a better parameter to mark stages of snail sexual maturity.

Among the selected Bgl-miRNAs in this study, bglmiR-8 (Fig. 4A) was up-regulated at 10 days compared to its level of expression at other times. At this age, the snail presents intense body growth, suggesting that bglmiR-8, somehow, regulates biological processes that regulate the development of the snail. Similarly, in Drosophila sp. miR-8 acts on the cell surveillance and epithelial organisation of various regulators of act in protein ${ }^{(37)}$ thus interfering with the process of homeostasis in the organism. ${ }^{(38)}$ These findings corroborate our targets prediction for this miRNA, which shows the regulation of processes like metabolism, response to stress and cell organisation [Supplementary data (Table III)], through silencing activity over gene targets including E3 ubiquitin-ligase, universal stress protein YxiE-like, TNF receptors, MAP kinases, and DNA-repair.

Bgl-miR-92 was significantly upregulated at 10 days and down-regulated at 20 days of snail development (Fig. 4B). It was predicted to regulate the response to stress and inflammatory process, by targeting such genes as leukocyte tyrosine kinase receptor-like and toll-like receptor [Supplementary data (Table III)]. Its ortholog in Crassostrea gigas was similarly shown to be involved in the control of inflammatory process by regulating the expression of tumor necrosis factor (TNF). ${ }^{(39)}$ This earlier finding and our results allow for prediction of bglmiR-92 function in regulating prominent immunological process in B. glabrata. Further, it is worth emphasising that in our study, the snail development was evaluated at an interval when snail presents intense cell proliferation, which was already recognised being regulated by miR92 in humans. ${ }^{(40)}$

The miR-184 showed differential expression relative to the stages of development with marked down-regulation of expression in snails of age 20 days (Fig. 4C). The targets found for bgl-miR-184 [Supplementary data 
(Table III)] corroborated with previous findings that orthologs act in the growth regulation, proliferation and, apoptosis of epithelial cells. ${ }^{(41,42,43,44)}$

Unlike the down regulation shown in reasonable number of miRNAs in the study, bgl-mi-315, bglmiR-375 and bgl-miR-750 showed up-regulation through all developmental stages of B. glabrata (Fig. 4D, F, G). This profile suggested that the targets for these miRNAs play significant role in B. glabrata development. However, further studies are required to establish their roles on the biology of the mollusc. The target prediction suggested important action on targets like bcl-2 and caspase-3 [Supplementary data (Table III)], well recognised as cell growth regulators. Orthologs of Bgl-miR-315 and bgl-miR-375 act by regulating the cell growth and differentiation in other organisms. ${ }^{(45,46)}$ Since miR-750 and miR-315 seem to play an important role in regulating the innate defense system of shrimp ${ }^{(47)}$ our results on targets prediction suggest it has a similar function in $B$. glabrata. We also predicted it as a likely regulator of the piRNAs efficiency in the snail, since it has Piwi and Tudor genes as targets, and ubiquitin-conjugating enzyme E2 D3 [Supplementary data (Table III)].

Bgl-miR-1984 (Fig. 4E) was intensely expressed at snail age 10 days. The expression was significantly higher than in other stages. The target prediction showed the regulation of genes related to microtubule activity and metabolic processes linked to immune response [Supplementary data (Table III)]. Orthologs of bgl-miR-8 and bgl-miR-1984 were closely related to the regulation of the immune response in C. gigas. ${ }^{(48)}$ This is suggestive of the involvement of miR-1984 in the biology of B. glabrata as it is one of the micro RNAs exclusively produced in mollusk. Expression assays using mollusks infected with $S$. mansoni may provide further information about the biological importance of this miRNA.

Through the results described above, the presence of miRNAs in B. glabrata was confirmed and their conceivable roles in the biology of the snail were predicted. It is worth mentioning that the existence of the small RNA biogenesis pathway was previously shown, as well as its expression outline in infected and non-infected snails. ${ }^{(7)}$ The recognition of a well-characterised system for post-transcriptional gene expression regulation system through the Bgl-miRNA opens opportunity for new studies that will aim to elucidate its role in the snail susceptibility to $S$. mansoni infection.

In the present study, we were also able to identify a broad repertoire of Bgl-piRNAs. The role of piRNAs is strongly linked to transposon silencing, epigenetic regulation, and control of mRNA stability in different organisms, ${ }^{(13)}$ including mollusks. ${ }^{(49)}$ Our data showed that the putative Bgl-piRNAs identified have strongly conserved uridine at 5 'ends (1U) and adenine at the tenth position (10A). It was also identified that most of the sense and antisense reads of Bgl-piRNAs presented an overlap at exactly ten nucleotides in B. glabrata genome. This is remarkably a ping-pong cycle signature, ${ }^{(12,13)}$ which shows that $B$. glabrata relies on this biogenesis pathway to generate its mature piRNAs that are able to act as gene expression regulators.
A higher diversity in putative Bgl-piRNAs in snail samples with shell demonstrates that the presence of hemolymph is a determinant to the enrichment of piRNAs repertoire. It is even more relevant since the overall number of aligned reads was higher in the sample without shell. It shows that, even with a smaller depth of sequencing, greater diversity of piRNAs sequences are present in the hemolymph when compared to that of snail tissues.

The relevance of the results in Bgl-piRNAs in B. glabrata corroborated with previous studies that showed the presence of piRNAs in circulating fluids of different organisms and are used as biomarkers for several disorders. (22) The repertoire of circulating Bgl-piRNAs probably regulates different aspects of the snail biology by interfering with a broad range of processes that will be explored in future studies. This is achievable as previous finding shave demonstrated the importance of hemolymph on $B$. glabrata defense against $S$. mansoni infection. . $^{(3,4,5,21)}$

In conclusion - This present study showed that B. glabrata presented a vast repertoire of miRNAs and piRNAs suggesting that these small RNAs probably interfere with snail biology throughout its life cycle, and act on the immune regulation of this organism. The molecules reported in this study have similar features as those of reference organisms and, present notable diversity, especially in B. glabrata hemolymph. The results open possibilities on studying the role of the small RNAs in the mollusk susceptibility to $S$. mansoni infection, as well as its development. This study also provided important information that can be explored for the development of new molluscicides. Additional studies (some already ongoing), involving silencing techniques, may contribute to elucidating functional roles of the small RNAs.

\section{ACKNOWLEDGEMENTS}

To the facility on real-time PCR, bioinformatic platform and Sequencing platform of the Instituto René Rachou, to the post-graduation program in health sciences of Instituto René Rachou/Fiocruz Minas Gerais. We are very grateful to Dr Oyetunde Timothy Oyeyemi for his valuable contribution to the improvement of the writing style of the text.

\section{AUTHORS' CONTRIBUTION}

MSG, RLC, LMS, EHB and FR. proposed the research; MSG, RLC, LMS, EHB, WJJ, LRA, LGP, PMZC and FRQ designed the experiments; MSG, RLC, EHB, WJJ, LGP, LRA and FRQ prepared samples, performed experiments, performed the statistical analyses and performed bioinformatic analysis; MSG, WJJ, LGP and FRQ wrote a manuscript and prepared figures; MSG, RLC, LMS, EHB and PMZC supervised the project. All authors reviewed the manuscript. The authors declare there is no conflict of interest, neither of financial or non-financial origin, involved in the publication of this article. The funders had no role in study design, data collection and analysis, decision to publish, or preparation of the manuscript.

\section{REFERENCES}

1. Paraense WL, Correa LR. Variation in susceptibility of populations of Australorbis glabratus to a strain of Schistosoma mansoni. Rev Inst Med Trop São Paulo. 1963; 5: 15-22.

2. Negrao-Correa D, Mattos AC, Pereira CA, Martins-Souza RL, 
Coelho PM. Interaction of Schistosoma mansoni sporocysts and hemocytes of biomphalaria. J. Parasitol Res. 2012; 2012: 743920.

3. Bayne CJ, Buckley PM, DeWan PC. Macrophage like hemocytes of resistant Biomphalaria glabrata are cytotoxic for sporocysts of Schistosoma mansoni in vitro. J. Parasitol. 1980; 66(3): 413-9.

4. Pereira CA, Martins-Souza RL, Correa Jr A, Coelho PM, NegraoCorrea D. Participation of cell-free haemolymph of Biomphalaria tenagophila in the defence mechanism against Schistosoma mansoni sporocysts. Parasite Immunol. 2008; 30(11-12): 610-9.

5. Granath Jr WO, Yoshino TP. Schistosoma mansoni: passive transfer of resistance by serum in the vector snail, Biomphalaria glabrata. Exp Parasitol. 1984; 58(2): 188-93.

6. Adema CM, Hillier LW, Jones CS, Loker ES, Knight M, Minx $\mathrm{P}$, et al. Whole genome analysis of a schistosomiasis-transmitting freshwater snail. Nat Commun. 2017; 8: 15451.

7. Queiroz FR, Silva LM, Jeremias WJ, Baba EH, Caldeira RL, Coelho PMZ, et al. Differential expression of small RNA pathway genes associated with the Biomphalaria glabrata/Schistosoma mansoni interaction. PLoS One. 2017; 12(7): e0181483.

8. de Souza Gomes M, Donoghue MT, Muniyappa M, Pereira RV, Guerra-Sa R, Spillane C. Computational identification and evolutionary relationships of the microRNA gene cluster miR-71/2 in protostomes. J Mol Evol. 2013; 76(6): 353-8.

9. de Souza Gomes M, Muniyappa MK, Carvalho SG, Guerra-Sa R, Spillane C. Genome-wide identification of novel microRNAs and their target genes in the human parasite Schistosoma mansoni. Genomics. 2011; 98(2): 96-111.

10. Simoes MC, Lee J, Djikeng A, Cerqueira GC, Zerlotini A, da Silva-Pereira RA, et al. Identification of Schistosoma mansoni microRNAs. BMC Genomics. 2011; 12: 47.

11. Moazed D. Small RNAs in transcriptional gene silencing and genome defense. Nature. 2009; 457(7228): 413-20.

12. Brennecke J, Stark A, Russell RB, Cohen SM. Principles of microRNA-target recognition. PLoS Biology. 2005; 3(3): e85.

13. Brennecke J, Aravin AA, Stark A, Dus M, Kellis M, Sachidanandam R, et al. Discrete small RNA-generating loci as master regulators of transposon activity in Drosophila. Cell. 2007; 128(6): 1089-103.

14. Aravin AA, Sachidanandam R, Bourc'his D, Schaefer C, Pezic $\mathrm{D}$, Toth KF, et al. A piRNA pathway primed by individual transposons is linked to de novo DNA methylation in mice. Mol Cell. 2008; 31(6): 785-99.

15. Girard A, Sachidanandam R, Hannon GJ, Carmell MA. A germline-specific class of small RNAs binds mammalian Piwi proteins. Nature. 2006; 442(7099): 199-202.

16. Malone CD, Brennecke J, Dus M, Stark A, McCombie WR, Sachidanandam R, et al. Specialized piRNA pathways act in germline and somatic tissues of the Drosophila ovary. Cell. 2009; 137(3): $522-35$

17. Rajasethupathy P, Antonov I, Sheridan R, Frey S, Sander C, Tuschl T, et al. A role for neuronal piRNAs in the epigenetic control of memory-related synaptic plasticity. Cell. 2012; 149(3): 693-707.

18. Huang X, Fejes Toth K, Aravin AA. piRNA Biogenesis in Drosophila melanogaster. Trends Genet. 2017; 33(11): 882-94.

19. Hayashi R, Schnabl J, Handler D, Mohn F, Ameres SL, Brennecke J. Genetic and mechanistic diversity of piRNA 3'-end formation. Nature. 2016; 539(7630): 588-92.

20. Livak KJ, Schmittgen TD. Analysis of relative gene expression data using real-time quantitative PCR and the 2(-Delta Delta C(T)) Method. Methods. 2001; 25(4): 402-8.
21. Bayne CJ, Buckley PM, DeWan PC. Schistosoma mansoni: cytotoxicity of hemocytes from susceptible snail hosts for sporocysts in plasma from resistant Biomphalaria glabrata. Exp Parasitol. 1980; 50(3): 409-16.

22. Vychytilova-Faltejskova P, Stitkovcova K, Radova L, Sachlova M, Kosarova Z, Slaba K, et al. Circulating PIWI-Interacting RNAs piR-5937 and piR-28876 are promising diagnostic biomarkers of colon cancer. Cancer Epidemiol Biomarkers Prev. 2018; 27(9): 1019-28.

23. Luan Y, Zhang S, Zuo L, Zhou L. Overexpression of miR-100 inhibits cell proliferation, migration, and chemosensitivity in human glioblastoma through FGFR3. Onco Targets Ther. 2015; 8: $3391-400$

24. Negi V, Paul D, Das S, Bajpai P, Singh S, Mukhopadhyay A, et al. Altered expression and editing of miRNA-100 regulates iTreg differentiation. Nucleic Acids Res. 2015; 43(16): 8057-65.

25. Gendron CM, Pletcher SD. MicroRNAs mir-184 and let-7 alter Drosophila metabolism and longevity. Aging Cell. 2017; 16(6): 1434-8.

26. Teixeira LK, Reed SI. Ubiquitin ligases and cell cycle control. Ann Rev Biochem. 2013; 82: 387-414.

27. Liu C, Zhang S, Wang Q, Zhang X. Tumor suppressor miR-1 inhibits tumor growth and metastasis by simultaneously targeting multiple genes. Oncotarget. 2017; 8(26): 42043-60.

28. Heidersbach A, Saxby C, Carver-Moore K, Huang Y, Ang YS, de Jong PJ, et al. microRNA-1 regulates sarcomere formation and suppresses smooth muscle gene expression in the mammalian heart. Elife. 2013; 2: e01323.

29. Zhu JY, Heidersbach A, Kathiriya IS, Garay BI, Ivey KN, Srivastava D, et al. The E3 ubiquitin ligase Nedd4/Nedd4L is directly regulated by microRNA 1. Development. 2017; 144(5): 866-75.

30. Wu YC, Lee KS, Song Y, Gehrke S, Lu B. The bantam microRNA acts through Numb to exert cell growth control and feedback regulation of Notch in tumor-forming stem cells in the Drosophila brain. PLoS Genet. 2017; 13(5): e1006785.

31. Chase R, Tolloczko B. Tracing neural pathways in snail olfaction: from the tip of the tentacles to the brain and beyond. Microse Res Tech. 1993; 24(3): 214-30.

32. Boulias K, Horvitz HR. The C. elegans microRNA mir-71 acts in neurons to promote germline-mediated longevity through regulation of DAF-16/FOXO. Cell Metab. 2012; 15(4): 439-50.

33. Yang G, Yang L, Zhao Z, Wang J, Zhang X. Signature miRNAs involved in the innate immunity of invertebrates. PLoS One. 2012; 7(6): e39015.

34. Huang T, Xu D, Zhang X. Characterization of host microRNAs that respond to DNA virus infection in a crustacean. BMC Genomics. 2012; 13: 159

35. Reinhart BJ, Slack FJ, Basson M, Pasquinelli AE, Bettinger JC, Rougvie AE, et al. The 21-nucleotide let-7 RNA regulates developmental timing in Caenorhabditis elegans. Nature. 2000; 403(6772): 901-6.

36. Jain S, Rana V, Tridibes A, Sunil S, Bhatnagar RK. Dynamic expression of miRNAs across immature and adult stages of the malaria mosquito Anopheles stephensi. Parasit Vectors. 2015; 8: 179.

37. Bolin K, Rachmaninoff N, Moncada K, Pula K, Kennell J, Buttitta L. miR-8 modulates cytoskeletal regulators to influence cell survival and epithelial organization in Drosophila wings. Dev Biol. 2016; 412(1): 83-98.

38. Lee GJ, Hyun S. Multiple targets of the microRNA miR-8 contribute to immune homeostasis in Drosophila. Developmental and comparative immunology. 2014; 45(2): 245-51. 
39. Chen H, Jiang S, Wang L, Wang L, Wang H, Qiu L, et al. Cgi-miR$92 \mathrm{~d}$ indirectly regulates TNF expression by targeting CDS region of lipopolysaccharide-induced TNF-alpha factor 3 (CgLITAF3) in oyster Crassostrea gigas. Fish Shellfish Immunol. 2016; 55: 577-84.

40. Manni I, Artuso S, Careccia S, Rizzo MG, Baserga R, Piaggio G, et al. The microRNA miR-92 increases proliferation of myeloid cells and by targeting p63 modulates the abundance of its isoforms. FASEB J. 2009; 23(11): 3957-66.

41. Bi X, Cao Y, Chen R, Liu C, Chen J, Min D. MicroRNA-184 promotes proliferation and inhibits apoptosis in $\mathrm{HaCaT}$ cells: an in vitro study. Med Sci Monit. 2016; 22: 3056-61.

42. Mansuri MS, Singh M, Begum R. miRNA signatures and transcriptional regulation of their target genes in vitiligo. J Dermatol Sci. 2016; 84(1): 50-8.

43. Liang XG, Meng WT, Hu LJ, Li L, Xing H, Xie G, et al. MicroRNA-184 modulates human central nervous system lymphoma cells growth and invasion by targeting iASPP. J Cell Biochem. 2017; 118(9): 2645-53.

44. Zhuang Y, Peng H, Mastej V, Chen W. MicroRNA regulation of endothelial junction proteins and clinical consequence. Mediators Inflamm. 2016; 2016: 5078627.
45. Silver SJ, Hagen JW, Okamura K, Perrimon N, Lai EC. Functional screening identifies miR-315 as a potent activator of Wingless signaling. Proc Natl Acad Sci USA. 2007; 104(46): 18151-6.

46. Zhang Q, Wang Y, Liang J, Tian Y, Zhang Y, Tao K. Bioinformatics analysis to identify the critical genes, microRNAs and long noncoding RNAs in melanoma. Medicine (Baltimore). 2017; 96(29): e7497.

47. Kaewkascholkul N, Somboonviwat K, Asakawa S, Hirono I, Tassanakajon A, Somboonwiwat K. Shrimp miRNAs regulate innate immune response against white spot syndrome virus infection. Dev Comp Immunol. 2016; 60: 191-201.

48. Zhou Z, Wang L, Song L, Liu R, Zhang H, Huang M, et al. The identification and characteristics of immune-related microRNAs in haemocytes of oyster Crassostrea gigas. PLoS One. 2014; 9(2): e88397.

49. Jehn J, Gebert D, Pipilescu F, Stern S, Kiefer JST, Hewel C, et al. PIWI genes and piRNAs are ubiquitously expressed in mollusks and show patterns of lineage-specific adaptation. Commun Biol. 2018; $1: 137$. 